\section{How To Get Some Action (In Photoshop)}

\author{
Jerry Sedgewick \\ University of Minnesota \\ sedge001@umn.edu
}

All of us who work with images understand the enormous amount of time post-processing can require. Before you know it, a few hours can easily slip by even for the most seasoned among us. When so many other things need to be done, it can be frustrating to spend hours on image preparation.

Fortunately, some more frequent steps taken in Photoshop can be automated, and these can be assigned to one of the Function keys ( $F$ keys) at the top of the keyboard, or a droplet can be made (Photoshop 6x \& 7x only).

At a particular workstation at our core facility in which a 12-bit, grayscale camera is used to acquire fluorescently labeled sections, the F3, F4, F5 or F6 key can be pushed to auto-convert the image into various colors and bit-depths. The image file is saved out of the software acquisition program as a 16-bit TIFF image. The image then needs to be scaled in Photoshop using Leve/s (and moving the white, input slider to the white end of the histogram), converted to 8 bit grayscale (when images are not going to be measured for optical densities or intensities), and then kept as grayscale or converted to RGB. When it is an RGB image, it is then made into red, green, or blue images, depending on the fluorochrome used (in Levels: click drop down to select red, green, or blue channel NOT wanted, and then move the black input slider to the right toward the white end of the histogram, not quite eliminating all the unwanted colors).

How to create actions. These steps were automated and assigned a Function Key using the Actions dialogue box in Photoshop. To open this dialogue box, first open the image you wish to work on. Under Window, choose Actions or Show Actions.

The default setting for the Actions dialogue box will include a file folder icon labeled Defauit Actions.atn. This file folder represents a set which then contains any number of actions. Each set or file folder, then, can be used to organize actions into logical groups. In order to make an action you will need to start by making a set. Click on the upper, right arrowhead in the Actions dialogue box, highlight Create Set and then give it a name.

Next, click on the arrowhead and highlight Create Action. Give that action a name. In that dialogue box you will also note that the

\section{Actions Dialogue Box}

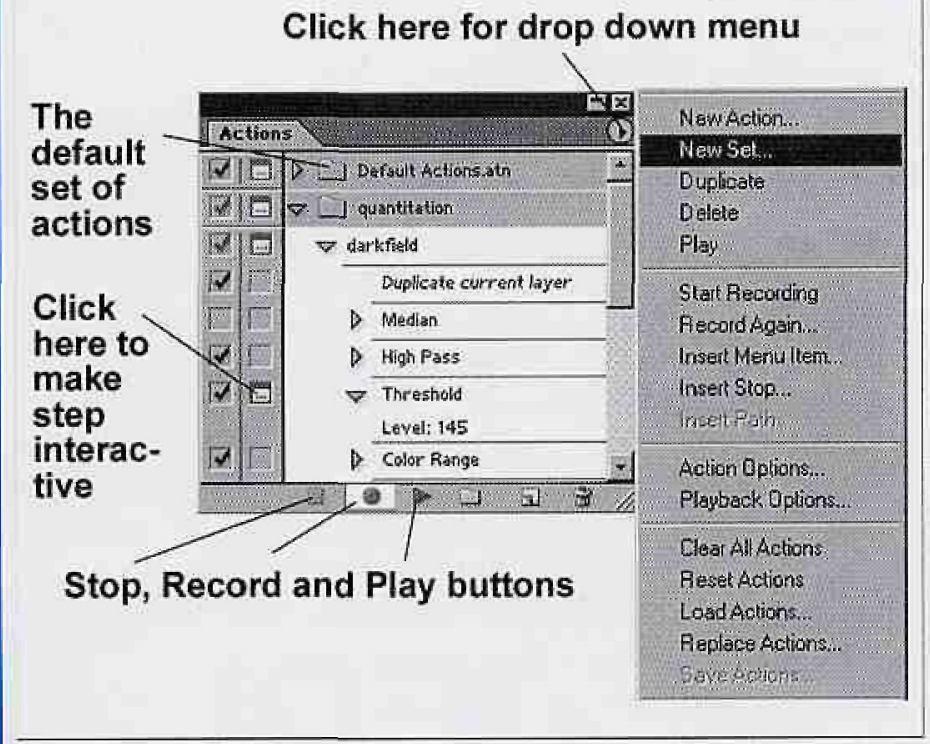

action can be given a Function key. Click that arrowhead and choose a key from the drop down menu. Click Record.

At this point, everything you do in Photoshop will be recorded. You will note that a round, red button at the bottom of the Actions dialogue box is pushed in. You can now run through your series of steps. If you need to first test the step before committing to recording it, you can always stop the recording by clicking on the black square button at the bottom of the Actions dialogue box. Test the action to see how it works, and then, if satisfied, Undo that step, click on the record button and repeat that step. If you make a mistake along the way, you can always stop the recording by clicking on the square, black key, highlight the step you wish to eliminate, and then either drag into the trash icon at the bottom of the dialogue box, or click on the arrowhead to choose Delete from the drop down menu. Again, you will have to Undo the last step and repeat it correctly when recording the subsequent time.

Once you are done, click the black, square button. Revert your image to when it was last saved by choosing Revert under File on the menu. Use the F key you have assigned to this action, or click on the action to highlight it and then click on the arrowhead at the bottom of the Actions dialogue box to run the series of steps. This should be done to test the steps.

Making Steps Interactive. You may want to make your steps interactive so that the user can make adjustments. We did that for users so that they could move the white input slider in the Levels dialogue box themselves. To do that, simply click in the box to the left of that step to create a white box icon.

Open and Save Steps: Automating for Several Files. Do not include steps which include opening the file or saving it (unless you wish to make an action to Save As in a different file format). Photoshop will only open and save that particular file if you do so (e.g., if you open a file named "Experiment1.tif, "then that action will only operate on "Experiment1.tif" and no other image files). If you want to apply the action to several files in the same folder, that is done by using the Automate/Batch command under File on the menu.

The Batch dialogue box allows you to choose the set and the action, and the folder of images to which you'd like to apply the action (called Source). The Override Action Open Command is only checked when you have mistakenly included an open command in your action. Choose a Destination by clicking the adjacent arrowhead to reveal a drop down box. Choose Save and Close, or, better yet, choose Folder (you will have to create a folder into which to place post-action images). Note that Photoshop $7 x$ may have some trouble finding folders that are placed in certain directories. This may just be a Windows issue, but I have noticed that folders are found when placed in the $\mathrm{C}$ : (root) directory one or two directories in.

If you do not make a choice for Destination, the default is None. In that instance, the action will take place on each image, but one image after another will stay on the desktop. That can lead to a computer crash.

In Photoshop $7 x$ additional features include the ability to rename files. This can be useful when image files need to maintain an order.

Droplets. In Photoshop $6 x$ and $7 x$, you can create what is called a droplet. Under File choose Automate/Create Droplet. You will be prompted for the Destination directory. The droplet becomes a shortcut to that action within Photoshop. It is useful to place the droplet (which takes the form of an icon) on the desktop so that folders with images can be dragged on the icon. Photoshop will automatically open and the action will be done on each image in that folder. That can be done versus using the Batch command, depending on your preference.

Troubleshooting. You will note that arrowheads lie next to each step in your action. By clicking on these arrowheads, you can 


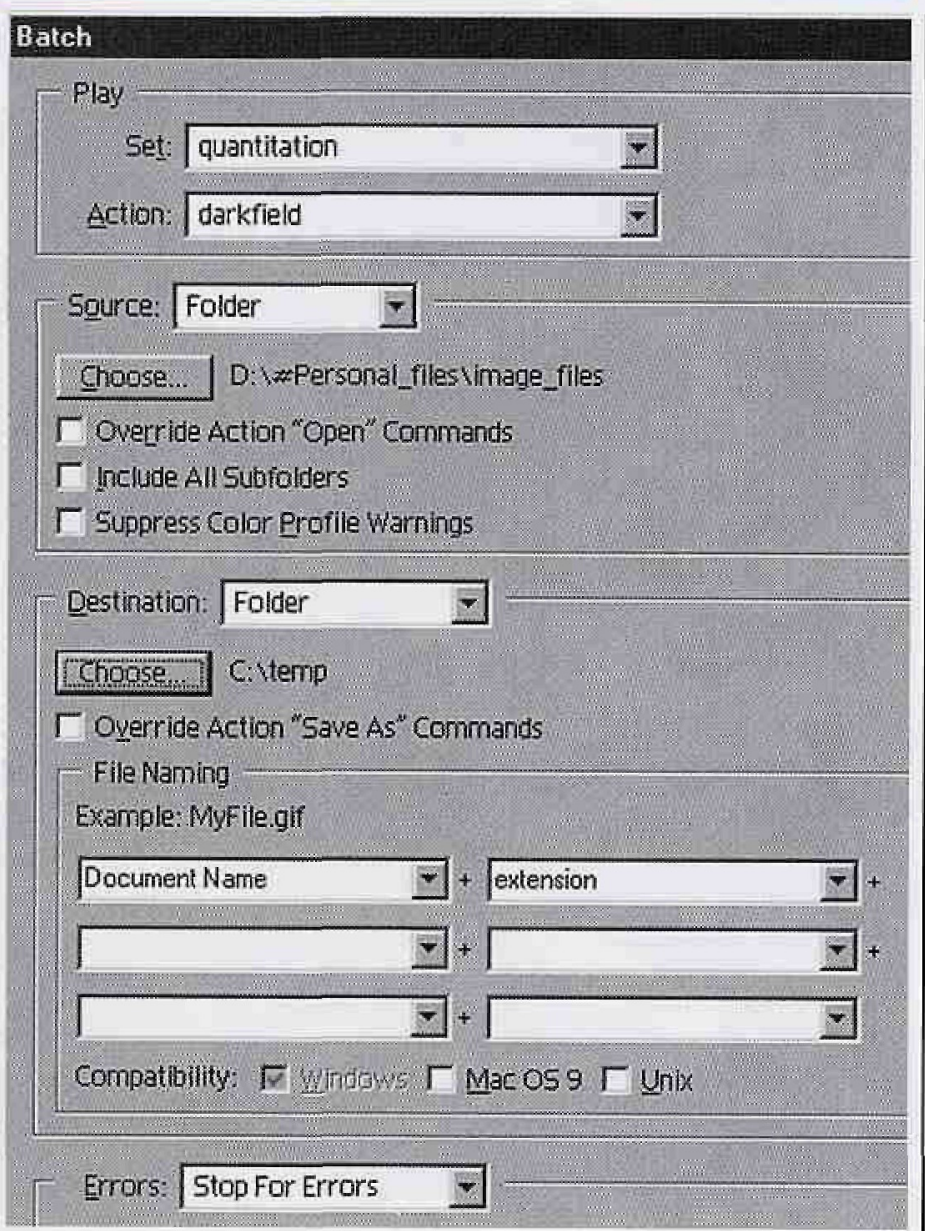

Batch Dialogue Box. Be sure to set the Source and Destination for image files before running actions on a folder of image filles.

see more information about each step. A review of this information can reveal mistakes in your procedure. Especially, when making mistakes while recording, stopping, starting and undoing along the way, actions can go awry. Often, it is best to start over, taking care not to make a mistake along the way.

You can also attempt too many steps in one action, or the action may involve more than one image. In those instances, it works best to break the actions up into smaller parts. Sometimes these parts can then be combined into one action by recording more than one batch automation, but that can complicate matters to the point at which new problems crop up.

Note that in Photoshop you cannot open an image from one folder and then from a different folder to run a series of steps (e.g. when combining images to look at colocalization). In that instance, it is best to place each pair or triplicate of images in separate folders to run actions. When working with separate images, it is best to paste one image over another so that you work, instead, with layers.

Saving and Loading Actions. To back up actions onto your computer or a floppy disk for sharing between computers, select the set you want and click on the upper, right arrowhead in the Actions dialogue box. Choose Save Actions. To load actions from a disk, click the arrow again and choose Load Actions from the drop down menu. Find the action you want to load and highlight Load.

Post a note of the Function Keys to which you've assigned actions and imaging work will never take nearly as much time or effort.

N.B.: Photoshop and Adobe are trademarks of the Adobe Corporation
International Association of Advisors for Electron Microscopy

\section{How good are results from your laboratory?}

\section{EM is a competitive world, can you survive? How do outsiders know of your competence?}

\section{Do you deserve accreditation?}

For more information

www.iaaem.com

or email

admin@iaaem.com

\section{NORTHERN

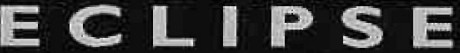

LINITED TINE OFFER

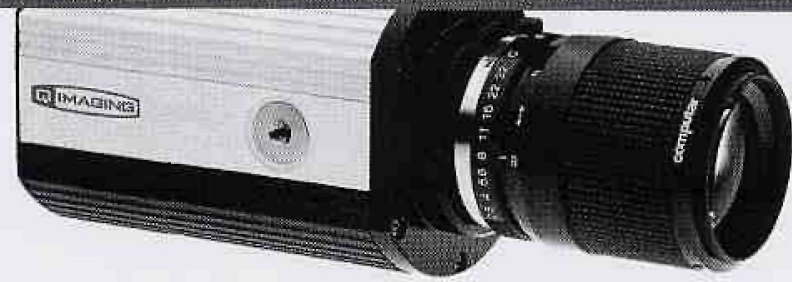

Get the power of Northern Eclipse 6.0 FREE by ordering a QImaging FireWire CCD camera When you buy a QImaging CCD FireWire camera directly from us at the manufacturer's suggested list price, we will include, for a limited time, one FREE Northern Eclipse 6.0 application of your choice. Applications such as Brightness/ Time, Time-Lapse, Image Merging, Fast Capture to Ram, and even De-convolution are available. See the On Sale page of our web site for a full list of applications available with this offer.

www.empix.com

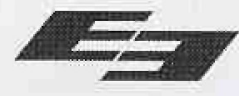

E M P I X 